\title{
PROJECT REPORT
}

Development of an occupational-therapy-led paediatric burn telehealth review clinic

\section{AUTHORS}

\section{O

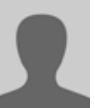 \\ Lauren Matheson² BOccThy (Hons), Occupational Therapist \\ Tilley Pain ${ }^{3}$ PhD, Principal Research Fellow \\ Gail A Kingston ${ }^{4} \mathrm{PhD}$, Assistant Director Occupational Therapy * \\ CORRESPONDENCE}

Debra Phillips ${ }^{1}$ BOccThy (Hons), Grad Cert Health Management, Occupational Therapist

*Dr Gail A Kingston gail.kingston@health.qld.gov.au

\section{AFFILIATIONS}

1, 2, 4 Occupational Therapy Department, The Townsville Hospital and Health Service, PO Box 670, Townsville, Qld 4810, Australia

${ }^{3}$ The Townsville Hospital and Health Service, PO Box 670, Townsville, Qld 4810, Australia; and College of Public Health, Medical and Veterinary Sciences, James Cook University, Townsville Qld 4811, Australia

\section{PUBLISHED}

16 August 2021 Volume 21 Issue 3

\section{HISTORY}

RECEIVED: 22 June 2020

REVISED: 23 November 2020

ACCEPTED: 1 February 2021

\section{CITATION}

Phillips D, Matheson L, Pain T, Kingston GA. Development of an occupational-therapy-led paediatric burn telehealth review clinic. Rural and Remote Health 2021; 21: 6223. https://doi.org/10.22605/RRH6223

This work is licensed under a Creative Commons Attribution 4.0 International Licence

\section{ABSTRACT:}

Context: Burns are a common injury in children. Rural and remote children with burn injuries are disadvantaged if their burns require hospitalisation and specialist rehabilitation. Most specialist burn rehabilitation is provided in regional or metropolitan cities by a multidisciplinary team. Therefore, rural and remote burn patients are required to travel to access these services. This project aimed to develop an Occupational Therapy (OT)-Led Paediatric Burn Telehealth Review Clinic (OTPB Clinic) at Townsville University 
Hospital (TUH) to provide ongoing rehabilitation to rural and remote children after burn injury closer to home.

Issues: Local audits identified inequitable service delivery to children from rural and remote areas after burn injury. A project officer was appointed to develop the OTPB Clinic, including comprehensive guidelines to support sustainability. An expanded scope role was undertaken by the treating OT, and allied health assistants were engaged to promote efficient service delivery.

Lessons learned: The OTPB Clinic commenced in 2017 and was evaluated using patient satisfaction surveys and number of clinical Keywords:

allied health, Australia, burns, paediatrics, scope of practice, telerehabilitation. encounters pre- and post-implementation. During the implementation period, 28 rural or remote paediatric burn patients were reviewed. Review frequency increased from 20-week to 8 -week intervals. Travel time was reduced by approximately 12 hours per appointment. Families identified numerous benefits of the clinic including continuity of care and reduced time away from work. Less than $4 \%$ of patients required re-engagement with paediatric surgeons for surgical intervention. The model has the potential to be transferred to other tertiary referral burns services.

\section{FULL ARTICLE:}

\section{Context}

This article describes the development of a new model of care (MOC) for the rehabilitation of rural and remote paediatric burns patients. The MOC was developed at Townsville University Hospital (TUH), a tertiary referral hospital in northern Queensland. Among TUH specialist services is the North Queensland Paediatric Burn Service (NQPBS) treating approximately 170 children a year who sustain burn injuries. Approximately $29 \%$ of the children treated by NQPBS reside in regional communities, $20 \%$ in remote or very remote communities across northern Queensland and the remainder from the Townsville region. The NQPBS is not a dedicated burns unit. Rather, it is a burns service within the paediatric ward at TUH, which also provides other specialty services.

Burns are a common injury in children. Deep-partial or fullthickness burns often require hospitalisation and surgical intervention followed by complicated post-surgical rehabilitation to achieve optimal functional performance, minimal scarring and full range of movement ${ }^{1}$. Optimal outcomes following severe burns are best achieved through specialist multidisciplinary team interventions. $^{2}$. Within these teams, occupational therapists (OTs) have a major role. OT interventions after burn injury include the management of hypertrophic scarring, joint range of motion, pain and functional ability ${ }^{2}$. Specialist OT burn services, located in tertiary referral hospitals, are rarely available in rural and remote areas. Thus, patients from rural and remote communities have limited access to these services ${ }^{\mathbf{3 , 4}}$.

Children from rural and remote areas have a higher likelihood of complications following burn injuries. Complications mean they may require longer length of stay in hospital due to the limited availability of burns resources, specialist knowledge and outreach services in rural areas ${ }^{3}$. An in-house audit of paediatric burns patients at TUH confirmed rural and remote burns patients received fewer reviews than their Townsville region counterparts. Therefore, the OT-Led Paediatric Burn Telehealth Review Clinic (OTPB Clinic) was developed with the aim of addressing the gap in access to burn rehabilitation services for rural and remote children.

The OTPB Clinic is an expanded scope model of care for the OT. In this model, the paediatric surgeon delegates the role of primary treating clinician to monitor and treat children undergoing active burn rehabilitation to the OT. Recommendations on expanded scope models of care, outlined by the Allied Health Professions Office of Queensland, were incorporated in the development of the model ${ }^{\mathbf{5}}$.

\section{Issues}

The model was developed to address some recurring issues that occur in rural health service delivery. First, an in-house audit was conducted to describe service delivery to rural and remote children with burn injuries. The audit revealed clear differences between Townsville and rural and remote children, with the latter having increased complications and receiving fewer follow-up services. The in-house audit conducted between July and December 2016 showed children from rural and remote areas were reviewed on average every 20 weeks by an occupational therapist. Comparatively, children with burn injuries living in Townsville were reviewed at 6-8-week intervals. A range of administrative, clinical, geographical, technical and workforce-related concerns contributed to this inequity (Table 1).

Innovative healthcare models for rural and remote residents often fail because of lack of procedures, guidelines and evaluation ${ }^{\mathbf{6 , 7}}$. To ameliorate this risk, an experienced OT was employed as the project officer via external funding obtained from the Allied Health Professional Office of Queensland. The dedicated time allowed the OT to develop the new model of care, which included comprehensive guidelines regarding all aspects of service delivery.

Implementing practice change in health services is complex. Therefore, an advisory committee was established consisting of paediatric surgeons, paediatric outreach nurses, a specialist OT in paediatric burns, remote-area OTs and telehealth coordinators. A literature search of previous paediatric burn telehealth services to examine salient design features for translation to the northern Queensland context was conducted ${ }^{\mathbf{8 - 1 1}}$. The committee developed service guidelines and pathways using an established e-Learning package ${ }^{\mathbf{1 2}}$, with the aim of improving access to burn rehabilitation for rural and remote children.

Eligibility criteria for the OTPB Clinic were developed: 
- undergone surgical intervention such as debridement, grafting or reconstruction because of their burn injury

- prolonged time to definitive wound healing ( $>14$ days) as documented in the medical record

- identified as high risk of scarring (eg family history of significant hypertrophic or keloid scarring, high-risk ethnicity groups, comorbidities that may be associated with significant scarring)

- ongoing monitoring required by NQPBS (eg patients requiring surgical intervention following dog bite injuries).

Patients were eligible for referral to the OTPB Clinic if they were a patient of the NQPBS and been reviewed by an NQPBS surgeon on at least one occasion.

A specialist OT, working in an expanded scope role, was the lead clinician for the burn review. Paediatric surgeons were no longer involved with clinical reviews unless children met clinical criteria. Clinical criteria for re-engaging the paediatric surgeon were developed by the advisory committee in case further surgical intervention such as reconstruction, releases and steroid injections were required (Table 2). A multidisciplinary approach was adopted for complex patients. This enabled joint telehealth consultations with nurses and/or physiotherapists to address issues such as ongoing wounds or reduced range of motion after complex burn injuries.

Pathways for burn rehabilitation were customised to individual family situations. Children within a 30-minute drive of TUH continued via the usual care pathway (ie face-to-face visits with the paediatric surgeon). Children discharged to hospitals visited by the NQPBS surgeons (Cairns and Mackay) were referred to their local OT including face-to-face review by the visiting surgeon. Children from rural and remote areas were reviewed via telehealth by the Townsville-based OTPB Clinic.
Family choice was incorporated into the service design and delivery. Therefore, a range of service models to deliver the OTPB Clinic were developed to account for differences in family access to telehealth and local health professionals. The OTPB Clinic uses existing telecommunications technology available in families' homes and includes an expansive network of telehealth technology in hospitals and primary health clinics in rural and remote sites. Appointments could be delivered to the family home using video-conferencing software (for smart devices) or the Queensland Health Telehealth Portal (on a household computer) using a collaborative meeting room. Infrastructure and hardware to support telehealth is highly variable in rural and remote areas, which meant that, for some families, home consultations were inaccessible. Alternatively, the family could choose to attend their local rural hospital, community health service or clinic to access Queensland Health telehealth services.

Review appointments in rural settings were scheduled to include local clinicians. The models to include local clinicians were the same profession, if a local OT was available, or a different profession (eg clinical nurse, Indigenous liaison officer or other allied health professional) if no local or visiting OT services were available ${ }^{\mathbf{1 2}}$. The local health professional assisted in several tasks for the review and in turn they were upskilled in burns rehabilitation by the OTPB Clinic's OT (Table 3). Clinical reviews were completed solely by the OTPB Clinic if no local services were available.

Efficient and timely service delivery was sought through the support of allied health assistants $(\mathrm{AHAs})^{\mathbf{1 3}}$. Delegation of clinical tasks to an AHA (Table 3) were developed using the Calderdale Framework ${ }^{\mathbf{1 4}, \mathbf{1 5}}$. Educating families to use the technology to enable home consultations was completed by the AHA prior to appointments.

Table 1: Root cause analysis of inequity of service delivery for paediatric burn injuries in rural and remote northern Queensland

\begin{tabular}{|l|l|}
\hline Cause & \multicolumn{1}{c|}{ Description } \\
\hline Administrative & $\begin{array}{l}\text { Lack of formal referral and booking processes for rural and remote patients } \\
\text { Time-consuming booking processes for telehealth consultations involving multiple } \\
\text { negotiations }\end{array}$ \\
\hline Clinical & $\begin{array}{l}\text { Duplication or loss to follow-up due to lack of coordination between the paediatric } \\
\text { surgeon appointments and OT reviews } \\
\text { Telehealth not prioritised or offered as an option, so patients had to attend busy } \\
\text { face-to-face clinics } \\
\text { Inadequate clinical knowledge prior to telehealth consultations therefore not } \\
\text { meeting the child's clinical need }\end{array}$ \\
\hline Geographical & Extensive travel required to access follow-up in Townsville \\
\hline Technology & Failed consultations due to technology issues \\
\hline Workforce & High turnover or lack of allied health staff in rural and remote communities \\
\hline \multicolumn{1}{|c|}{ OT, occupational therapist. }
\end{tabular}


Table 2: Clinical criteria for re-engagement with paediatric surgeon

- Contracture

- Wound breakdown or skin shortening /contracture

- Persistent pruritus that may benefit from pharmacological intervention

- A scar deemed as not responding to standard non-invasive scar management interventions 6 months beyond injury (to account for timeframes of hypertrophic scar development) as demonstrated by worsening Patient Observer Scar Assessment Scale or patient/caregiver concerns

- Patient reported functional limitations and pain when engaging in routine occupations

- Request by parent or patient

- Upon cessation of active scar management. The OTPB Clinic has not been designed to provide long-term surveillance for children at risk of needing reconstructive surgery and hence the need for at-risk patients to be re-engaged with NQPBS surgeons.

Table 3: Tasks performed by community occupational therapist or delegated to alternative health professional to assist Occupational Therapist-Led Paediatric Burn Telehealth Review Clinic

\begin{tabular}{|l|l|}
\hline Community health professional tasks & \multicolumn{1}{|c|}{ Allied health assistant tasks } \\
\hline Assisting children to demonstrate range of motion & Coordinate telehealth booking with host and \\
Assisting families to manipulate garments and/or & recipient sites \\
splints for the occupational therapist at the host & Check quality of telehealth connection prior to \\
site to review fit & appointment (if in patient's home) \\
Palpating scar tissue to assist the occupational & Obtain information via a pre-telehealth phone call \\
therapist at the host site to appreciate the scar & utilising a structured script \\
vascularity, pigmentation and pliability & Request a digital still photograph of the scar \\
Capture and forward digital still photograph to & Complete outcome measure (Brisbane Burns Scar \\
OTPB Clinic & Impact Profile) (ref. 9) \\
\hline
\end{tabular}

OTPB, Occupational Therapist-Led Paediatric Bum Telehealth Review.

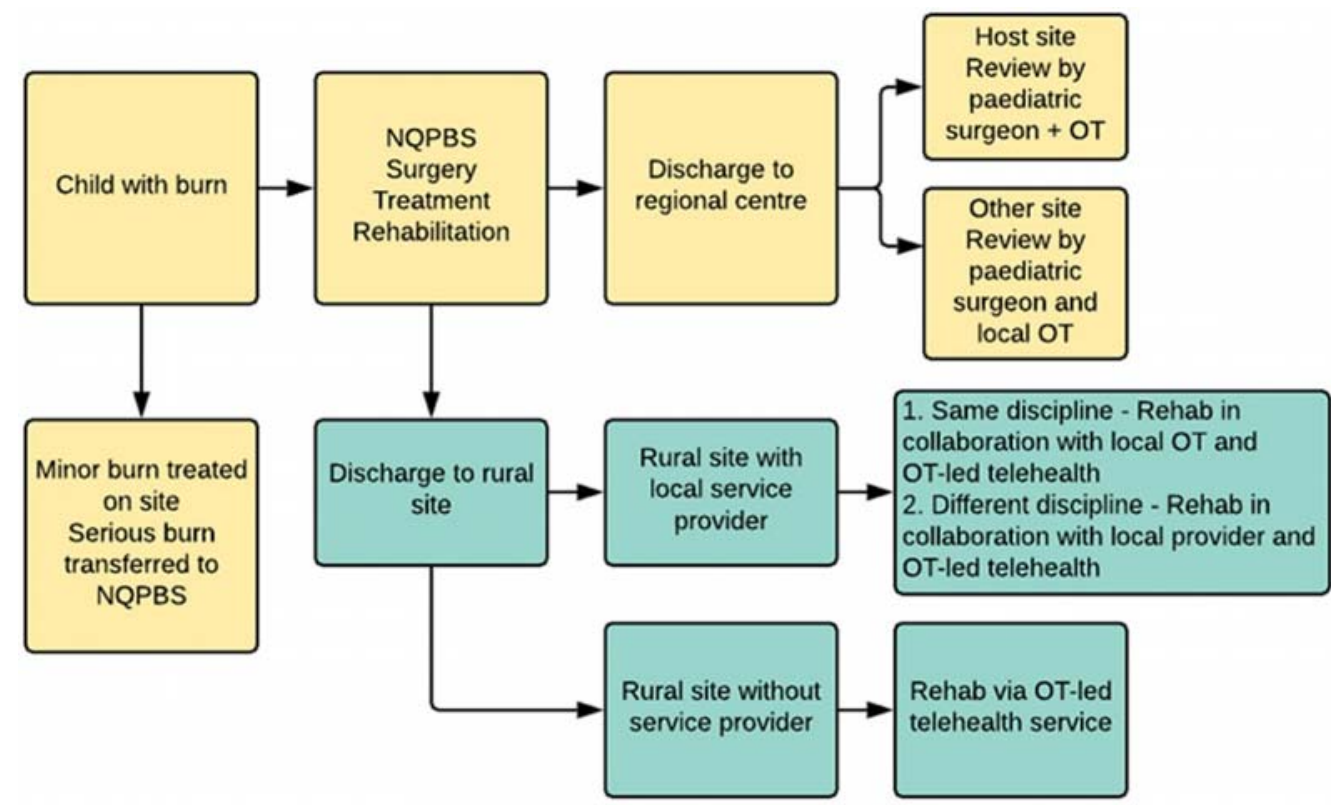

${ }^{t}$ Yellow denotes usual care and green denotes new OTPB model of care for those living in a rural or remote setting. OT, occupational therapist. NQPBS, North Queensland Paediatric Burn Service. OTPB, Occupational Therapist-Led Paediatric Burn Telehealth Review.

Figure 1: Model-of-care pathway for children with burns in northern Queensland. ${ }^{\dagger}$

\section{Ethics approval}

Evaluation of this model of care was endorsed by the Townsville Hospital and Health Service Human Research Ethics Committee (reference number HREC/17/QTHS/147).

\section{Lessons learned}

A concurrent evaluation was planned alongside the development of the clinic. Quantitative and qualitative data were collected to measure effectiveness and acceptability of the OTPB Clinic. Data were collected by the project officer via the integrated electronic medical record (ieMR), and a survey specifically designed for this service evaluation was conducted by an AHA.

Twenty-eight children attended the clinic between January and June 2017. Each child received between one and three consultations for a total of 76 occasions of service for the period. Children from rural and remote settings were reviewed on average every 8 weeks during the trial phase, representing a substantial 
increase from the period prior to the introduction of the service. The OT led the clinic, which meant that 76 occasions of service were released for the paediatric surgeon to see other children on the waiting list. A median travel time of 12 hours per consultation was saved for the families. Travel time savings were calculated from the published duration of commercial transport, drive times when commercial transport was not available or a combination of both, depending on the location of the patient.

Survey responses completed by families involved in the trial phase were positive. The survey consisted of 17 questions, including open questions asking respondents to state advantages and disadvantages of the new model of care. Advantages included time saved, added convenience because of not travelling to TUH for appointments, reduced need for caregivers to take time off work, and children did not miss school. Families valued the continuity of having the same knowledgeable therapist involved for the duration of their child's care, positive interactions between the child and therapist, and the support local clinicians received during the reviews. Disadvantages included difficulty in assessing the thickness of the child's scar when compared to a face-to-face appointment.

During the trial period, one child required surgical review ( $<4 \%$ of patients). Re-engagement of the child to the paediatric surgeon was initiated by the OT performing the OTPB Clinic review as per clinical criteria for re-engagement.

There are numerous benefits to the OTPB Clinic model. It embraces multidisciplinary collaboration and integrated care across the continuum to aid recovery and improve outcomes ${ }^{\mathbf{1 6}}$ by including rural clinicians in the telehealth review where possible. By delegating tasks to an AHA, the OT was able to work successfully in an expanded scope role ${ }^{\mathbf{1 7}}$. Additional benefits are likely to accrue to patients and the health service ${ }^{\mathbf{1 8}}$ because of reduced transport $\operatorname{costs}^{\mathbf{1 9}}$. Despite the demonstrated benefits, further evaluation of the OTPB Clinic is recommended. A larger evaluation incorporating more participants and multiple sites would provide greater insight into the success of the model. An evaluation of the access, cost and outcomes of children undergoing burns rehabilitation through the OTPB clinic is planned. A qualitative research study is underway to explore the experiences of families and clinicians who have interfaced with the OTPB Clinic.

In summary, a new model of care has been developed for the follow-up of rural and remote children after burn injury via telehealth. The model includes an expanded scope role for the OT, freeing up appointments for paediatric surgeons to see patients on their waiting lists. To date the model has successfully increased the frequency of follow-up appointments for rural and remote paediatric patients and is well received by families, and rural and remote health service staff. This model can be translated to tertiary burn services in other health services.

\section{Acknowledgements}

Funding from the Allied Health Professions Office of Queensland supported planning, implementation and evaluation of the OTPB Clinic at TUH.

\section{REFERENCES:}

1 Edgar D (Ed.). Burn trauma rehabilitation: allied health practice guidelines. Philadelphia, PA: Lippincott Williams \& Wilkins, 2014.

2 Simons M, King S, Edgar D. Occupational therapy and physiotherapy for the patient with burns: principles and management guidelines. Journal of Burn Care Research 2003;

24(5): 323-335. DOI link

3 Hyland E, Zeni G, Harvey J, Holland A. Rural and metropolitan pediatric burns in New South Wales and the Australian Capital Territory: does distance make a difference? Journal of Burn Care Research 2015; 36(4): 231-237. DOI link

4 Mills A, Millsteed J. Retention: an unresolved workforce issue affecting rural occupational therapy services. Australian Occupational Therapy Journal 2002; 49(4): 170-181. DOI link

5 Allied Health Professions' Office of Queensland. Allied Health Professions' Office of Queensland. Ministerial Taskforce on health practitioner expanded scope of practice: final report. Brisbane: Queensland Health, 2014.

6 Tham R, Humphreys J, Kinsman L, Buykx P, Asaid A, Tuohey K. Study protocol: evaluating the impact of a rural Australian primary health care service on rural health. BMC Health Services Research 2011; 11(1): 52. DOI link

7 Wakerman J, Humphreys JS. Sustainable primary health care services in rural and remote areas: innovation and evidence.

\section{Australian Journal of Rural Health 2011; 19(3): 118-124. DOI link}

8 Smith AC, Garner L, Caffery LJ, McBride CA. A review of the paediatric telehealth for pre- and post-operative surgical patients. Journal of Telemedicine and Telecare 2014; 20(7): 400-404. DOI link

9 McWilliams T, Gilroy F, Wood FM. The successes and challenges of providing paediatric burns service by telehealth in Western Australia. Journal of Telemedicine and Telecare 2007; 13(3): 63-64. DOI link

10 Xu C, Wotton R, Gramotnev H, Kimble RM. A randomized controlled trial of supplementary digital imaging with real-time telemedicine in post-acute burns care of children. Journal of Telemedicine and Telecare 2007; 13(3): 109-110.

11 Turk E, Karagulle E, Aydogan C, Ogus O, Tarim A, Karakayali,H, et al. Use of telemedicine and telephone consultation in decision making and follow-up of burn patients: initial experience from two burn units. Burns 2011; 37: 415-419. DOI link

12 Allied Health Professions' Office of Queensland. Allied Health Telehealth Education package. Brisbane: Queensland Health, 2016.

13 Allied Health Professions' Office of Queensland. Allied Health Assistant Framework. Brisbane: Queensland Health, 2015.

14 Stute M, Hurwood A, Hulcombe J, Kuipers P. Pilot implementation of allied health assistant roles within publicly funded health services in Queensland, Australia: results of a 
workplace audit. BMC Health Services Research 2014; 14(1): 1. DOI link

15 Stute M, Hurwood A, Hulcombe J, Kuipers P. Defining the role and scope of practice of allied health assistants within Queensland public health services. Australian Health Review 2013; 37(5): 602-606. DOI link

16 Kornhaber R, Rickard G, McLean L, Wiechula R, Lopez V, Cleary M. Burn care and rehabilitation in Australia: health professionals' perspectives. Disability and Rehabilitation 2019; 41(6): 714-719. DOI link
17 Young G, Hulcombe J, Hurwood A, Nancarrow S. The Queensland Health Ministerial Taskforce on health practitioners' expanded scope of practice: consultation findings. Australian Health Review 2015; 39(3): 249-254. DOI link

18 Smith AC, Scuffham P, Wootton R. The costs and potential savings of a novel telepaediatric service in Queensland. BMC Health Services Research 2007; 7: 35. DOI link

19 McWilliams T, Hendricks J, Twigg D, Wood F, Giles M. Telehealth for paediatric burn patients in rural areas: a retrospective audit of activity and cost savings. Burns 2016; 42(7): 1487-1493. DOI link

This PDF has been produced for your convenience. Always refer to the live site https://www.rrh.org.au/journal/article/6223 for the Version of Record. 